\title{
Possible Association between SNAP-25 Single Nucleotide Polymorphisms and Alterations of Categorical Fluency and Functional MRI Parameters in Alzheimer's Disease
}

\author{
Franca Rosa Guerini ${ }^{\mathrm{a}, *}$, Cristina Agliardi ${ }^{\mathrm{a}}$, Manuela Sironi ${ }^{\mathrm{b}},{\text { Beatrice } \text { Arosio }^{\mathrm{c}} \text {, Elena Calabrese }}^{\mathrm{a}}$, \\ Milena Zanzottera ${ }^{\mathrm{a}}$, Elisabetta Bolognesi ${ }^{\mathrm{a}}$, Cristian Ricci ${ }^{\mathrm{a}}$,, , Andrea Saul Costa ${ }^{\mathrm{a}}$, Daniela Galimberti ${ }^{\mathrm{d}}$, \\ Ludovica Griffanti ${ }^{\text {a,e }}$, Anna Bianchi ${ }^{\mathrm{a}}$, Federica Savazzi ${ }^{\mathrm{a}}$, Daniela Mari ${ }^{\mathrm{c}}$, Elio Scarpini ${ }^{\mathrm{d}}$, \\ Francesca Baglio $^{\mathrm{a}}$, Raffaello Nemni ${ }^{\mathrm{a}, \mathrm{f}}$ and Mario Clerici ${ }^{\mathrm{a}, \mathrm{g}}$ \\ ${ }^{a}$ Don C. Gnocchi Foundation IRCCS, Milano, Italy \\ ${ }^{\mathrm{b}}$ Scientific Institute IRCCS E. Medea, Bosisio Parini (LC), Italy \\ 'Geriatric Unit, Department of Clinical Sciences and Community, University of Milano, Fondazione Ca' Granda, \\ IRCCS Ospedale Maggiore Policlinico, Milano, Italy \\ ${ }^{\mathrm{d}}$ Dino Ferrari Center, Department of Pathophysiology and Transplantation, University of Milano, Fondazione Ca' \\ Granda, IRCCS Ospedale Maggiore Policlinico, Milano, Italy \\ ${ }^{\mathrm{e}}$ Bioengineering Department, Politecnico of Milano, Milano, Italy \\ ${ }^{\mathrm{f}}$ Chair of Neurology, Department of Pathophysiology and Transplantation University of Milano, Milano, Italy \\ ${ }^{\mathrm{g}}$ Chair of Immunology, Department of Pathophysiology and Transplantation University of Milano, Milano, Italy \\ ${ }^{\mathrm{h}}$ Department of Epidemiology and Preventative Medicine, University of Regensburg, Regensburg, Germany
}

Handling Associate Editor: Marco Bozzali 


\section{INTRODUCTION}

The major cause of cognitive decline in the elderly is Alzheimer's disease (AD), a neurodegenerative disorder characterized by intracellular neurofibrillary tangles and extracellular amyloid $\beta$-peptide $(\mathrm{A} \beta)$ plaques. The pathogenesis of $\mathrm{AD}$ is complex and involves genetic factors that are still mostly unidentified and whose role is ill defined. Thus, the presence of the $\varepsilon 4$ allele of apolipoprotein E gene (APOE4), the only known confirmed genetic risk factor for sporadic $\mathrm{AD}$ is neither necessary nor sufficient for disease manifestation [1].

A consistent finding in AD is synaptic loss, and synaptic abnormalities are suggested to be the pathologic finding more closely correlated with this disease [2] and to play a primary role in the characteristic cognitive deficits seen in AD. This could be mediated by the loss of synapses itself or by an altered exocytosis of neurotransmitters, proteins stored in synaptic vesicles that are released into the synaptic cleft [3]. Several SNARE proteins are involved in exocytosis [4]; synaptosomal-associated protein of $25 \mathrm{kDa}$ (SNAP-25), in particular, is a vesicular SNARE protein playing an important role in the release of neurotransmitters. Thus, SNAP-25 interacts with voltage-gated calcium channels [5] inhibiting their function and reducing responsiveness to depolarization $[6,7]$. Changes in SNAP-25 levels are present in schizophrenia, with levels of the protein being decreased in the hippocampus and the frontal lobe Broadman's area (BA) 10, and increased in prefrontal lobe BA 9 and in the cingulate cortex [8] as well as in cerebrospinal fluid (CSF) [9]. A SNAP-25 promoter variant was also found to result in an augmented protein expression in the brain of patients with earlyonset bipolar disorders [10]. Higher levels of this protein have also been involved in attention deficit hyperactivity disorder [11], a condition characterized by hyperactive behavior and impaired attentive ability resulting in social dysfunction [12], and neuroticism [13]. In this case, the observed association is between attention deficit hyperactivity disorder and two single nucleotide polymorphism (SNPs) localized between intron 3 and the $3^{\prime}$ untranslated region of SNAP-25 gene (rs3746544 and rs1051312) [14, 15], whereas a third SNP (rs363043) correlates with hyperactive behavior in autistic children [16]. Notably, the SNAP-25 gene lies in an area linked to intelligence (20p12-p11.2) [17], and a family-based genetic association test performed in children and adults showed that particular SNAP-25 SNPs (rs363043, rs353016, rs363039, rs363050) are associated with variation in Intelligence Quotient (IQ) phenotypes. These SNPs are localized within intron 1 in a region spanning about $13.8 \mathrm{~kb}$, and are known to affect transcription factor binding sites [18].

In this study, we verified a possible involvement of SNAP-25 by comparing AD patients with ageand gender-matched healthy controls. In particular, the frequency of distribution of five SNAP-25 gene polymorphisms (rs363043, rs363039, rs363050, rs3746544, rs 1051312) was correlated with the degree of cognitive impairment evaluated with an extensive neuropsychological assessment. We considered a category fluency task as an indirect measure of long-term memory status (for a review, see [19]) allowing us to test verbal competences that rely on the structure of semantic network. Semantic fluency requires integrity of semantic concepts, and dysfunction occurs early in $\mathrm{AD}$ and causes significant disability with AD progression. Recently, evidences from neuropsychological $[20,21]$ and functional magnetic resonance imaging (fMRI) studies [22-24] showed that language deficits, especially those interesting verbal fluency functioning, are precursors of $\mathrm{AD}$ clinical condition.

Moreover, we replicated our findings in subjects with amnestic mild cognitive impairment (aMCI) and possible associations between SNAP-25 SNPs and disease status, degree of cognitive impairment and fMRI parameters were analyzed.

\section{MATERIALS AND METHODS}

\section{Ethical committee approvals}

Written consent was obtained from all the participants in the study or from their care givers. The consent was in accordance with the declaration of Helsinki; the ethical committees of the Don C. Gnocchi Foundation and of the Fondazione $\mathrm{Ca}^{\prime}$ Granda, IRCCS 
Table 1

Baseline characteristic of patients with a diagnosis of Alzheimer's disease (AD) or amnestic mild cognitive impairment (aMCI); healthy controls $(\mathrm{HC} 1$ and $\mathrm{HC} 2)$ are also included

\begin{tabular}{|c|c|c|c|c|}
\hline & $\mathrm{AD}(n=607)$ & $\mathrm{HC} 1(n=615)$ & $\mathrm{aMCI}(n=148)$ & $\mathrm{HC} 2(n=310)$ \\
\hline \multicolumn{5}{|l|}{ Parameters } \\
\hline Age $(y)[$ Mean \pm SD] & $76.7 \pm 8.2$ & $72.0 \pm 6.9$ & $76.8 \pm 7.8$ & $69.9 \pm 6.3$ \\
\hline Range (y) & 53-96 & 44-104 & 59-96 & $41-86$ \\
\hline Male $\%$ & 32.3 & 31.7 & 46.0 & 44.7 \\
\hline MMSE score $[$ mean $\pm \mathrm{SD}]$ & $18.2 \pm 6.5$ & $27.1 \pm 3.1$ & $25.2 \pm 1.3$ & $27.2 \pm 2.8$ \\
\hline Formal education $(y)[$ mean $\pm \mathrm{SD}]$ & $8.0 \pm 4.1$ & $8.1 \pm 3.8$ & $7.7 \pm 3.4$ & $7.9 \pm 3.9$ \\
\hline APOE $4+\%$ & $47.0^{1}$ & $19.0^{1 .}$ & $36.5^{2}$ & $22.2^{2}$ \\
\hline
\end{tabular}

APOE4+: positivity for epslion4 allele SD: standard deviation. ${ }^{1} \mathrm{AD}$ versus $\mathrm{HC} 1: p<0.001 \mathrm{OR}: 3.78 \mathrm{IC}(95 \%): 2.09-4.93 ;{ }^{2}$ aMCI versus $\mathrm{HC} 2$ : $p<0.001$ OR: $2.00 \mathrm{IC}(95 \%): 1.30-3.08$.

Ospedale Maggiore Policlinico in Milano approved the study.

\section{Patients and controls}

A total of 1680 Italians of Caucasian origin were enrolled; the study was designed as a case control comparing $607 \mathrm{AD}$ patients and 615 healthy controls (HC1). A replication study was conducted enrolling 148 patients with aMCI and a second group of 310 $\mathrm{HC}$ (HC2); both $\mathrm{HC} 1$ and $\mathrm{HC} 2$ were age-and gendermatched with the patients (Table 1). Patients were consecutively recruited by the Neurology Departments of the Don C. Gnocchi Foundation and of the Fondazione Ca' Granda, IRCCS Ospedale Maggiore Policlinico in Milano.

AD patients had a clinical diagnosis of probable $\mathrm{AD}$ in mild to moderate stage of disease according to the NINCDS-ADRDA Work Group criteria [25] and DSM IV-R [26]. Reversible causes of dementia were excluded after medical and neurological evaluation, laboratory analysis, CT scan or MRI, and other investigations when necessary (e.g., electroencephalography, single-photon emission computerized tomography scan, CSF examination, etc.); all AD cases were sporadic. Outpatients diagnosed with aMCI according to Petersen criteria [27] were consecutively recruited as well from those attending the Memory Disorders Outpatients Service of the Don Gnocchi Foundation. Only aMCI individuals considered at high risk to develop AD were enrolled in the study. To be eligible, aMCI subjects had to meet the following operational criteria: memory complaint, confirmed by an informant; abnormal memory function, documented by extensive neuropsychological evaluation; normal general cognitive function, as determined by both Clinical Dementia Rating (CDR) [28] scale (CDR with at least a 0.5 in the memory domain); no impairment in functional activities of daily living as determined by a clinical interview with the patient and informant; no significant cerebral vascular disease (Hachinski score less than or equal to 4) [29]; no major psychiatric illnesses with particular attention to exclude subjects with history of depression (Hamilton Depression Rating Scale score less than or equal to 12) [30, 31]. Patients are followed with annual brain MRI and routine laboratory tests, and re-evaluated approximately every 6 months with neurological examination and a battery of neuropsychological tests and scales.

Two groups of $\mathrm{HC}$ : $\mathrm{HC} 1$ and $\mathrm{HC} 2$ of unrelated Italians that were age- and gender-matched with AD and aMCI patients respectively were recruited as well. These individuals were selected according to the SENIEUR protocol for immuno-gerontological studies $[32,33]$; their cognitive status was assessed by Mini-Mental State Examination (MMSE) and mean raw data scores were reported in Table 1.

\section{Neuropsychological evaluation and psychometric assessment}

A randomly selected subgroup of 209 AD (72 males, 137 females) and 54 aMCI ( 24 males, 30 females) individuals underwent extensive neuropsychological evaluation that included MMSE [34], language functions tests (phonological and categorical fluency [35] and Token tests [36]), short-term memory tests (Corsi, Digit Span Forward and Backward tests [37]), longterm memory tests (Rey's Figure Delayed Recall [37]; Paired-Associate Learning test, and Story Recall test [35]) and frontal-executive functions (Raven Coloured Progressive Matrices [38]), visuo-spatial abilities (Rey's Figure Copy [37]). Categorical fluency impairment was also evaluated with fMRI verbal fluency task (the paced overt version of verbal fluency paradigm described by Basho and colleagues [39], see data analysis section). All the evaluation values were adjusted for age and educational level (conversion formulae are 
reported in the appropriate references) and only the corrected scores were used for correlation analysis.

\section{SNPs typing}

Genomic DNA was isolated from peripheral blood mononuclear cells by phenol-chloroform extraction. SNPs were typed using the Taqman SNP Genotyping Assays (Applied Biosystems by Life Technologies, Foster City, CA, USA) on an ABI PRISM 7000 Sequence Detection System. For rs363039, rs363043, rs363050 and rs3746544, respectively, the C_327976_10, C_2488346_10, C_329097_10, and C_27494002_10. Human Pre-Designed Assays (Applied Biosystems by Life Technologies) were used. The restriction enzyme polymorphism rs 1051312 was genotyped by DdeI digestion as previously described [14].

\section{APOE4}

Customer-designed taqman probes for the 112 and 158 codons were used. Primers and probes for the 112 codon are: 112 Forward primer: 5'-GGG CGC GGA CAT GGA G-3', 112 Reverse primer: $3^{\prime}-\mathrm{TCC}$ TCG GTG CTC TGG CC-5' 112 Arg Probe : 5'-CGT GCG CGG CCG-3'-FAM, 112 Cys Probe: 5'-ACG TGT GCG GCC GCC TG-3'-VIC. Primers and probes for the 158 codon are: 158 Forward primer: $5^{\prime}-\mathrm{TCC}$ GCG ATG CCG ATG-3', 158 Reverse primer: $3^{\prime}$-GCT CGG CGC CCT CG-5', 158 Arg probe: 5' -CCT GCA GAA GCG CCT GGC A-3'-FAM, 158 Cys probe: $5^{\prime}$-CCT GCA GAA GGG CCT GGG AGT-3'-VIC.

\section{fMRI protocol and data analysis}

MRI scans were obtained using a 1.5 Tesla scanner (Magnetom Avanto, Siemens, Erlangen, Germany). Functional images were acquired with single-shot gradient echo EPI sequence $(\mathrm{TR} / \mathrm{TE}=3000 / 50 \mathrm{~ms}$, voxel size $=3.9 \times 3.9 \times 3 \mathrm{~mm}^{3}, 38$ axial slices, 120 volumes $)$ using blood oxygenation level dependent (BOLD) contrast. A morphological three-dimensional T1-weighted MPRAGE sequence $(\mathrm{TR} / \mathrm{TE}=1900 / 3.37 \mathrm{~ms}$, voxel size $=1 \times 1 \times 1 \mathrm{~mm}^{3}$, number of axial slices $=176$ ) was also acquired to be used as anatomical scan for fMRI analysis.

Thirty-eight subjects (28 AD patients and $10 \mathrm{HC}$ ) were selected to perform the paced overt version of verbal fluency paradigm (ABAB block design) [40]. Eighteen of these patients carried the rs363050 (AA or AG) and rs363043 (CT or TT) genotype (group 1); the other 10 carried the rs363050 (GG) and rs363043 (CC) genotype (group 0). For each individual 6 semantic categories were randomly presented during the fMRI acquisition. Overt responses were obtained via an MRI-compatible patient response and sound system (VisuaStim Digital, Resonance Technology Inc.) The use of ePrime software (e-Prime 2.0 Psychology Software Tool, http://www.pstnet. com) ensured exact timing of prompts.

\section{Statistical analysis}

Chi-square analysis was used to exclude any deviation of SNP genotype distribution from Hardy -Weinberg equilibrium (HWE) and to compare casecontrol differences of SNPs distributions after gender stratification.

Haplotype analyses were performed using the SHEsis software freely available at http://202.120.7.14 /analysis/myAnalysis.php [40, 41]. The KolmogorovSmirnov (K-S) test was applied to verify normal distribution of numerical variable scores. Cognitive scores, which resulted normally distributed, were shown as mean and standard deviation (SD) and analysis of variance ANOVA was performed in relationship with SNPs distribution. For those variables, which were not normally distributed, Kruskall-Wallis test was applied. For genotype analyses $\mathrm{p}_{\mathrm{c}}$ values were corrected for 2 degree of freedom (degree of freedom of the genotype distribution of the three different genotypes); $p$ values of allelic comparison have only 1 degree of freedom because they analyze bialleic polymorphisms, therefore they did not need to be corrected for degree of freedom.

A multivariate logistic forward stepwise regression model corrected by gender and APOE4 positivity was used. This model had categorical fluency scores $<25$ or $>25$ as response variables in $\mathrm{AD}$ and in aMCI and genotype (rs363050 (AA/AG versus GG) and rs363043 (CT/TT versus CC)) as explanatory covariates, Post hoc power analysis were performed for all logistic regression and reported if the actual power was lower than $90 \%$.

Haplotype association analysis was performed using plink [42] by logistic regression; haplotype probabilities of individual subjects were incorporated as covariates in the regression model, which estimate the Odds ratios and $p$ values associated with having a score of categorical fluency impairment $\leq 25$, adjusting for gender and APOE4 positivity.

Statistical analysis on fMRI data was performed using SPM8 (SPM8, http://www.fil.ion.ucl. 
ac.uk/spm). Preprocessing of functional images involved realignment, co-registration to the anatomical image, spatial normalization to the Montreal Neurological Institute (MNI) space, and spatial smoothing with a $8 \mathrm{~mm}$ full-with at half-maximum (FWHM) Gaussian kernel. Single subjects statistical analysis was then performed with general linear model (GLM) approach [43] to detect the activation areas during the task (tcontrast: categorical fluency A versus control condition B). The contrast images obtained at the single-subject level were used to compute the second level analyses. An ANOVA full factorial design was employed with a 3 level factor to model the groups (AD grO, AD gr1, and HC), and with task performance, intracranial volume (ICV) (obtained by adding up white matter volume + grey matter volume + CSF volume) and MMSE scores as nuisance covariates, to adjust for potential confounds. We used a t-Student's contrast to assess the main effect of categorical fluency versus control condition in $\mathrm{HC}$ and $\mathrm{AD}$ (both gr0 and gr1) and to describe the difference among the genotypes (HC versus $\mathrm{AD}$ gr0; $\mathrm{HC}$ versus $\mathrm{AD}$ gr1; $\mathrm{AD}$ gr0 versus $\mathrm{AD}$ gr1). The maps resulting from the second level analyses were thresholded with two approaches: first, the activation clusters that survived after correction for multiple comparisons (Family wise correction, pFWEcorr $<0.05$ ) and an extent threshold of voxel size of 10 adjacent voxels were considered; then, for exploratory purposes, group differences were also described considering an uncorrected $p<0.001$ threshold with 50 or more contiguous voxels.

The contrast maps (one for each subject) entered in a second level analysis (ANOVA). ANOVA was performed to describe the difference among the three groups (HC, group 0, Group 1). Only activation clusters that survived after correction for multiple comparisons (Family wise correction, pFWE-corr <0.05) and an extent threshold of voxel size of 10 adjacent voxels were considered in the results section. Task performance entered the fMRI second level statistical analyses as a covariate due to the different performance obtained from AD (99\%) and HC (78\%).

\section{RESULTS}

SNAP-25 polymorphisms distribution

The five SNAP-25 gene SNPs were in Hardy Weinberg equilibrium in patients and controls, as shown by molecular genotyping. Genotype and allelic distribution comparisons revealed the presence of a significant association between $\mathrm{rs} 363050$ (AA) and
$\mathrm{AD}\left(\mathrm{p}_{\mathrm{c}}=0.002\right.$, OR:1.47) (Table 2a). Allelic distribution analyses confirmed that the rs363050 (A) allele is more frequently present in $\mathrm{AD}$ patients compared to $\mathrm{HC} 1(p=0.01, \mathrm{OR}: 1.24)$ and showed that the rs 363043 (T) allele is statistically more frequent as well in $\mathrm{AD}$ than in $\mathrm{HC1}(p=0.01$, OR:1.29). After stratification for gender, the rs363050 (AA) genotype and (A) allele resulted to be statistically more frequent in $\mathrm{AD}$ female patients compared to $\mathrm{HC} 1$ of the same gender $\left(\mathrm{p}_{\mathrm{c}}=0.001\right.$, OR:1.64 and $p=0.0003$, OR:1.46 respectively). Similarly, the $\operatorname{rs} 363043(\mathrm{~T})$ allele was statistically more frequent in female $\mathrm{AD}$ patients than in controls $(p=0.02$, OR:1.29). No significant differences were seen in males, possibly because of the lower analyzed numbers (Table $2 \mathrm{a}$ ).

The rs363050 (A) allele and the rs363043 (T) allele were also significantly associated with aMCI when these patients were compared with $\mathrm{HC} 2(p=0.01$, OR:1.42 and $p=0.04$, OR:1.35) (Table 2b). Because the replication cohorts of aMCI and controls were relatively small (148 versus 310$)$, the post hoc power of the $\mathrm{OR}$ estimate $\left(\mathrm{H}_{0} \mathrm{OR}=1\right)$ was calculated. Considering the size of the cohorts, an OR point estimate of 1.39 and frequencies of 34.9 and $28 \%$ respectively, the actual power was 0.814 .

The $\varepsilon 4$ allele of $A P O E 4$ is the only known confirmed genetic risk factor for sporadic AD. All the individuals were thus genotyped for APOE4 polymorphism and, as expected, results confirmed the presence of a positive association between APOE4 and both AD $(p<0.001$, OR: 3.78 versus HC1) and $\mathrm{aMCI}(p<0.001$, OR: 2.00 versus HC2) (Table 1). SNAP-25 genotypes were equally distributed in $A P O E 4+$ and $A P O E 4$ - individuals, indicating that $S N A P-25$ SNPs correlate with $\mathrm{AD}$ and aMCI independently of APOE4 (data not shown). Haplotype analysis of SNAP-25 SNPs and $A P O E$ evidenced a linkage disequilibrium between rs363050 and rs363043 and between rs3746544 and rs1051312 (Fig. 1), with the rs363050/rs363043 A$\mathrm{T}$ haplotype being statistically more frequent in $\mathrm{AD}$ compared to HC ( $p=0.002$, OR: 1.45$)$ and in aMCI compared to HC2 ( $p=0.03$, OR: 1.35$)$.

Finally no association was detected between the SNAP-25 SNPs (rs363039 (G/A), rs3746544 (T/G), and rs1051312 (T/C)) and either AD or aMCI (Supplementary Table 1a, b).

\section{SNAP-25 polymorphisms and neuropsychological evaluation}

Because the results above suggest an association between SNAP-25 genotypes and both $\mathrm{AD}$ and aMCI 
Table 2a

Genotype and allele distribution of the rs363050 and rs363043 SNAP-25 SNPs in Alzheimer's disease (AD) patients and in age- and gendermatched healthy controls ( $\mathrm{HC} 1$ ). Only results obtained in females are shown in details, as no significant differences were observed in male patients. OR: Odds ratio; 95\%CI: Interval of confidence. $\mathrm{p}_{\mathrm{c}}: p$ value corrected for two degree of freedom for genotype distribution

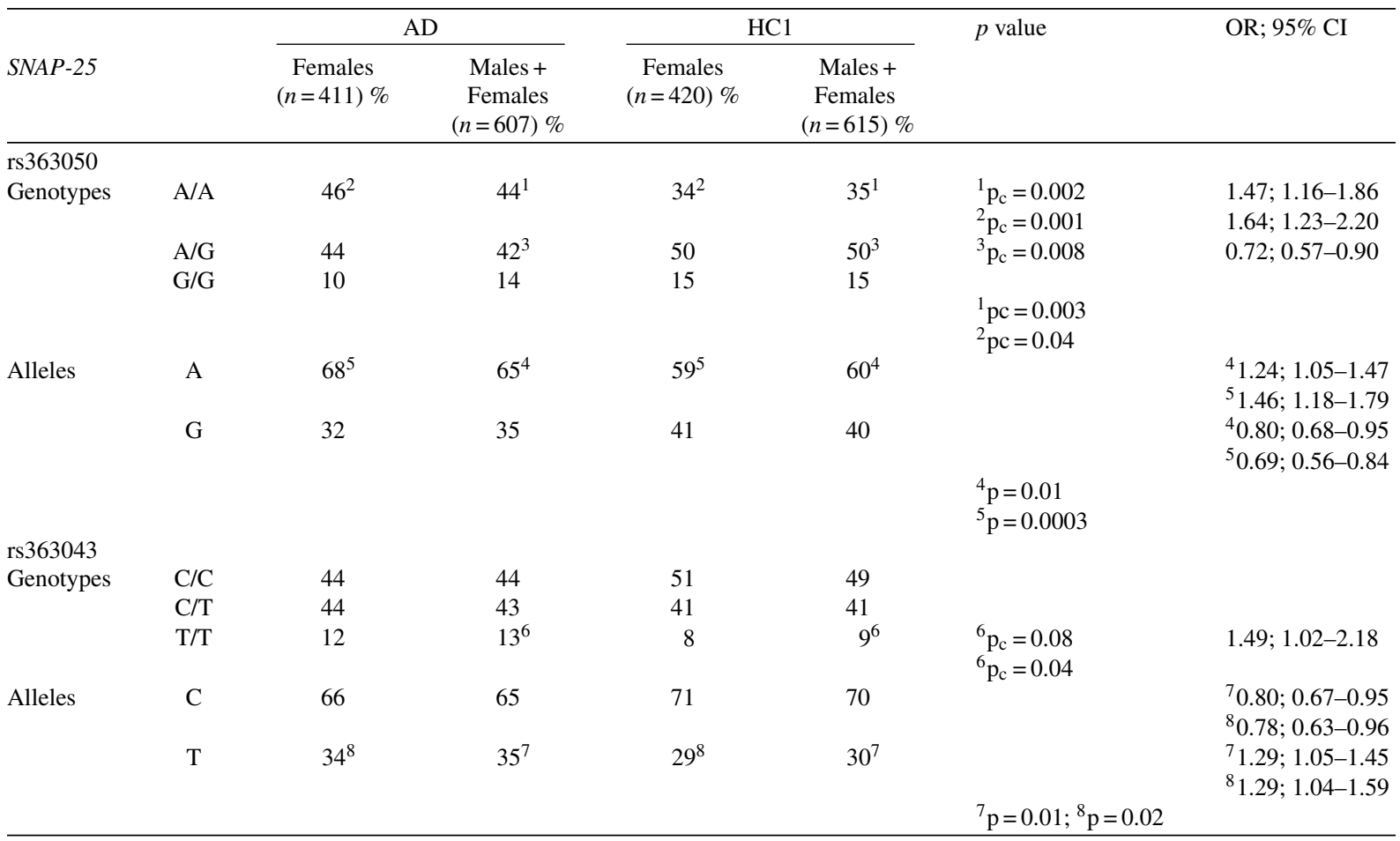

${ }^{1} \mathrm{p}_{\mathrm{c}}$ : AD versus $\mathrm{HC} 1 ;{ }^{2} \mathrm{p}_{\mathrm{c}}$ : female AD patients versus female $\mathrm{HC} 1 ;{ }^{3} \mathrm{p}_{\mathrm{c}}$ : AD versus $\mathrm{HC} 1 ;{ }^{4} \mathrm{p}$ AD versus $\mathrm{HC} 1 ;{ }^{5} \mathrm{p}$ : female AD versus female $\mathrm{HC} 1$; ${ }^{6} \mathrm{p}_{\mathrm{c}}$ : AD versus $\mathrm{HC} 1 ;{ }^{7} \mathrm{p}$ : AD versus $\mathrm{HC} 1 ;{ }^{8} \mathrm{p}$ : female AD versus female $\mathrm{HC} 1$.

Table $2 \mathrm{~b}$

Genotype and allele distribution of the rs363050 and rs363043 SNAP-25 SNPs in amnestic mild cognitive impairment (aMCI) patients and in age- and gender-matched healthy controls (HC2). OR: Odds ratio; $95 \%$ CI: Interval of confidence; $\mathrm{p}_{\mathrm{c}}$ : $p$ value corrected for two degree of freedom for genotype distribution

\begin{tabular}{|c|c|c|c|c|c|}
\hline \multirow[t]{2}{*}{ SNAP-25 } & & $\mathrm{aMCI}$ & $\mathrm{HC} 2$ & \multirow[t]{2}{*}{$p$ value } & \multirow[t]{2}{*}{ OR; 95\% CI } \\
\hline \multirow{2}{*}{\multicolumn{6}{|c|}{ rs363050 }} \\
\hline & & & & & \\
\hline \multirow[t]{3}{*}{ Genotypes } & $\mathrm{A} / \mathrm{A}$ & 39 & 30 & & \\
\hline & $\mathrm{A} / \mathrm{G}$ & 50 & 51 & & \\
\hline & $\mathrm{G} / \mathrm{G}$ & $11^{1}$ & $19^{1}$ & $\begin{array}{l}{ }^{1} \mathrm{p}_{\mathrm{c}}=0.04 \\
{ }^{1} \mathrm{pc}=0.04\end{array}$ & $0.52 ; 0.28-0.94$ \\
\hline \multirow[t]{3}{*}{ Alleles } & A & $64^{2}$ & $60^{2}$ & & ${ }^{2} 1.42 ; 1.07-1.90$ \\
\hline & $\mathrm{G}$ & 36 & 40 & & ${ }^{2} 0.70 ; 0.52-0.94$ \\
\hline & & & & ${ }^{2} \mathrm{p}=0.01$ & \\
\hline \multicolumn{6}{|l|}{ rs363043 } \\
\hline \multirow[t]{4}{*}{ Genotypes } & $\mathrm{C} / \mathrm{C}$ & 43 & 49 & & \\
\hline & $\mathrm{C} / \mathrm{T}$ & 45 & 41 & & \\
\hline & $\mathrm{T} / \mathrm{T}$ & 12 & 9 & & \\
\hline & & & & ${ }^{3} \mathrm{p}_{\mathrm{c}}=0.09$ & \\
\hline \multirow[t]{3}{*}{ Alleles } & $\mathrm{C}$ & 65 & 70 & & ${ }^{4} 0,73 ; 0,54-0,99$ \\
\hline & $\mathrm{T}$ & $35^{4}$ & $30^{4}$ & & ${ }^{4} 1,35 ; 1,01-1,82$ \\
\hline & & & & ${ }^{4} p=0.04$ & \\
\hline
\end{tabular}

${ }^{1} \mathrm{p}_{\mathrm{c}}$ : aMCI versus $\mathrm{HC} 2 ;{ }^{2} \mathrm{p}$ : aMCI versus $\mathrm{HC} 2 ;{ }^{3} \mathrm{p}_{\mathrm{c}}$ : aMCI versus $\mathrm{HC} 2 ;{ }^{4} \mathrm{p}$ : aMCI versus $\mathrm{HC} 2$. 


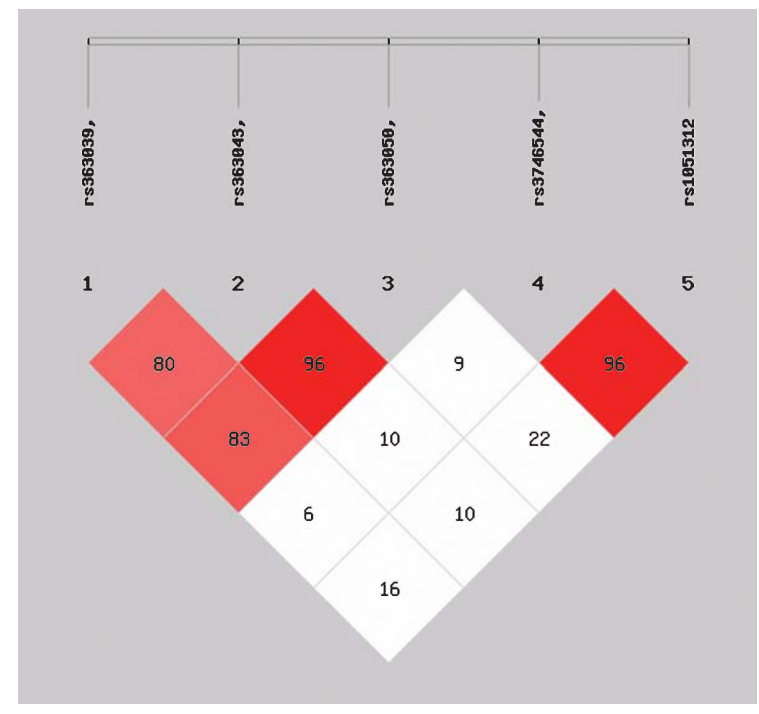

Fig. 1. LD pattern of $\left(\mathrm{r}^{2}\right)$ for the five selected SNPs within the SNAP-25 gene on chromosome 20 p12-p11.2.SNPs. (1) rs363039, (2) rs363043, (3) rs363050, (4) rs3746544, (5) rs1051312.

independently of APOE4, we verified possible correlations between such genotypes and $\mathrm{AD}$-associated clinical parameters in a randomly selected subset of $209 \mathrm{AD}$ and 54 aMCI patients. ANOVA analysis regarding categorical fluency scores showed that the rs363050 and rs363043 genotypes resulted as pathological $(\leq 25)$ in AD (df:2, F=4.65, $p=0.01$ and df:2, $\mathrm{F}=3.85, p=0.03$, respectively) (Table 3 ). Further supporting the importance of these SNPs in modulation of categorical fluency, data indicated that the cognitive skewing between pathological and normal value score increased when $\mathrm{AD}$ were stratified according to the presence/absence of the rs363050 (A) and rs363043 (T) alleles. Therefore lower categorical fluency scores were detected in patients carrying the rs363050 (A) allele in either homozygote rs363050 (AA) or heterozygote (AG) compared to those carrying rs 363050 (GG) (df:1, F=9.52, $p=0.002$ and df:1, F=7.54, $p=0.01$ ). Similarly, reduced categorical fluency scores were seen in rs363043 (TT) homozygote AD patients compared to those carrying the rs363043 (CC) genotype (df:1, F = 5.36, $p=0.02$ ). Notably, the skewing of categorical fluency scores was lower in patients carrying the rs363043 (CT) heterozygous pattern compared to those carrying the rs363043 (CC) genotype (df:1, $F=3.75, p=0.05$ ) (Table 3). Finally, no correlations were detected between SNAP-25 genotypes and categorical fluency scores in aMCI, in whom such scores were above the 25 points cut-off.

The relative contribution of $\mathrm{rs} 363050$ (AA/AG) versus rs363050 (GG) and of rs363043 (TT/CT) versus rs363043 (CC) in the model was evaluated next in a multivariate binary logistic regression model taking in account APOE4 positivity and gender as covariates. Categorical fluency scores $\leq 25$ or $>25$ were adopted as the response variable; SNAP-

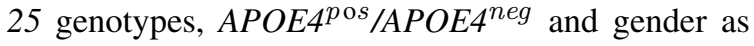
covariates. Stepwise binary logistic regression analysis evidenced that both rs363050 (AA/AG) and rs363043 (TT/CT) are statistically correlated to categorical fluency impairment when they are inserted singularly in regression analysis and adjusted for APOE4 and gender (rs363050 (AA/AG) $p=0.005$; OR: 3.93) (Table 4; Model 1); (rs363043 (TT/CT) $p=0.04$ OR: 1.82) (Table 4; Model 2). When both these variables and their interaction were evaluated in the same model, rs363050 (AA/AG) alone remained associated to categorical fluency impairment ( $p=0.01$ OR: 3.167). These results suggest that, even if both rs363050 (A) and rs363043 (T) alleles correlate with categorical flu-

Table 3

Categorical Fluency and SNAP-25 polymorphisms in AD and aMCI patients. SD, standard deviation, df, degree of freedom, $p, p$ value corrected for degree of freedom

\begin{tabular}{|c|c|c|c|c|c|c|c|c|c|c|c|c|c|}
\hline \multirow[b]{2}{*}{ SNAP-25 } & & \multicolumn{6}{|c|}{$\mathrm{AD}$} & \multicolumn{6}{|c|}{ aMCI } \\
\hline & & Mean & $\mathrm{n}$ & SD & $\mathrm{f}$ & df & $p$ & Mean & $\mathrm{n}$ & SD & f & df & $p$ \\
\hline \multicolumn{14}{|l|}{ rs363050 } \\
\hline \multirow[t]{4}{*}{ Genotypes } & $\mathrm{A} / \mathrm{A}$ & $24.2^{1}$ & 110 & 8.3 & ${ }^{1} 9.5$ & 1 & 0.002 & 33.3 & 20 & 8.7 & & & \\
\hline & $\mathrm{A} / \mathrm{G}$ & $24.3^{2}$ & 74 & 8.9 & 27.5 & 1 & 0.01 & 33.3 & 25 & 8.9 & & & \\
\hline & $\mathrm{G} / \mathrm{G}$ & $29.7^{1,2}$ & 25 & 6.8 & & & & 33.6 & 9 & 6.1 & & & \\
\hline & Total & 24.9 & 209 & 8.5 & 4.65 & 2 & 0.01 & 33.4 & 54 & 8.3 & & & n.s \\
\hline \multicolumn{14}{|l|}{ rs363043 } \\
\hline \multirow[t]{4}{*}{ Genotypes } & $\mathrm{C} / \mathrm{C}$ & $26.7^{3}$ & 82 & 9.0 & ${ }^{3} 5.4$ & 1 & 0.02 & 34.7 & 27 & 7.9 & & & \\
\hline & $\mathrm{C} / \mathrm{T}$ & $24.1^{4}$ & 97 & 8.4 & ${ }^{4} 7.5$ & 1 & 0.05 & 31.6 & 24 & 8.5 & & & \\
\hline & $\mathrm{T} / \mathrm{T}$ & $25.5^{3,4}$ & 30 & 6.1 & & & & 36.0 & 3 & 8.5 & & & \\
\hline & Total & $24.9^{3,4}$ & 209 & 8.5 & 3.85 & 2 & 0.03 & 33.4 & 54 & 8.3 & & & n.s. \\
\hline
\end{tabular}

${ }^{1} \mathrm{AA}$ versus GG; ${ }^{2} \mathrm{AG}$ versus GG; ${ }^{3} \mathrm{CC}$ versus TT; ${ }^{4} \mathrm{CT}$ versus TT. 
Table 4

Categorical Fluency and SNAP-25 polymorphisms in AD patients. Results of multivariate stepwise logistic regression analysis. Responsible variable: Categorical Fluency Score categorized as $\leq 25$ (pathological) or $>25$ (normal). OR, odds ratio; $95 \%$ CI, interval of confidence

\begin{tabular}{|c|c|c|c|c|c|}
\hline & & wald & $p$ value & OR & $95 \% \mathrm{CI}$ \\
\hline Model 1 & & 2.16 & 0.141 & 0.804 & $0.60-1.07$ \\
\hline Selected Variables & rs363050 (AA/AG) & 7.79 & 0.005 & 3.93 & $1.50-10.32$ \\
\hline Unselected Variables & $\begin{array}{l}\text { APOE } 4^{\mathrm{pos}} \\
\text { Gender }\end{array}$ & & $\begin{array}{l}0.58 \\
0.45\end{array}$ & & \\
\hline Model 2 & & 2.82 & 0.093 & 0.74 & $0.52-1.05$ \\
\hline Selected Variables & rs363043 (TT/CT) & 4.33 & 0.04 & 1.82 & $1.04-3.18$ \\
\hline Unselected Variables & $A P O E 4^{\mathrm{pos}}$ & & 0.72 & & \\
\hline & Gender & & 0.60 & & \\
\hline
\end{tabular}

Model 1: AD patients $(n=209)$; covariates: $A P O E 4^{p \text { os }}$ (e4/e4 e4/e3 e2/e4), Gender (female versus male), SNAP-25 rs363050 (AA/AG versus GG). Model 2: AD patients $(n=209)$; covariates: $A P O E 4^{p o s}$ (e4/e4 e4/e3 e2/e4), Gender (female versus male), SNAP-25 rs363043(TT/CT versus $\mathrm{CC}$ ).

Table 5

Logistic regression analysis by plink software adjusting for gender and $A P O E 4$ positivity. Categorical Fluency and SNAP-25 haplotype rs363050/rs363043 polymorphisms in AD patients. Responsible variable: Categorical Fluency Score categorized as $\leq 25$ (pathological) or >25 (normal); covariates: APOE $^{p \text { os }}$ (e4/e4 e4/e3 e2/e4), Gender (female versus male) OR: odds ratio

\begin{tabular}{lcclll}
\hline SNP1 & SNP2 & haplotype & wald & $p$ value & OR \\
\hline rs363050 & rs363043 & AT & 4.84 & 0.0278 & 1.25 \\
& & GC & 1.95 & 0.163 & 0.87 \\
& & AC & 0.562 & 0.454 & 0.923 \\
\hline
\end{tabular}

ency impairment, the involvement of rs363050 (A) is stronger than the one of rs363043 (T). Therefore, when analyzed together, one masks the other.

Finally, logistic regression analysis was performed using the Plink software to evaluate haplotype distribution in relationship with categorical fluency impairment $(\leq 25$ or $>25)$ and adjusting for gender and APOE4 positivity. Results confirmed that the rs363050/rs363043 A-T haplotype is significantly associated with lower categorical fluency scores $(p=0.03$ OR: 1.25) (Table 5).

\section{SNAP-25 and functional MRI}

To verify possible correlations between SNAP-25 genotypes and imaging patterns, a group of $\mathrm{AD}$ patients and $\mathrm{HC} 1$ underwent fMRI evaluation. Ten HC1, $18 \mathrm{AD}$ patients in whom the SNAP-25 SNPs being more frequent in $\mathrm{AD}$ were present (group 1), and $10 \mathrm{AD}$ patients in whom the SNAP-25 SNPs being more frequent in AD were NOT present (group 0) were analyzed. After discarding 4 patients (3 out of group 1 and 1 to out of group 0 ) for excessive motion artifacts, significant differences (ANOVA $p<0.001$ ) emerged. Bonferroni-corrected ANOVA results indicated that task performance was significantly better in $\mathrm{HC} 1$ (mean task performance 99.24\% $\pm 1.07 \%$ ) compared to $\mathrm{AD}$ patients (mean group $0: 76.37 \% \pm 12.91 \%$, $p=0.002$; mean group $1: 80.14 \% \pm 4.37 \%, p<0.001$ ); no significant differences were found between the two AD groups. Demographical, neuropsychological and behavioral task-fMRI characteristics were shown in Table 6.

Imaging results for category-driven word generation showed an activation in the bilateral (left $>$ right) frontal cortex (inferior and middle frontal gyri), the left premotor cortex, the bilateral cingulate gyrus, the medial temporal lobe cortex, and the basal ganglia in $\mathrm{HC} 1$. The overall pattern of brain activation seen in $\mathrm{AD}$ was similar to that seen in $\mathrm{HC} 1$, even if a significantly reduced recruitment was detected in the frontal cortex (Fig. 2A, B). Raising the statistical thresh-

Table 6

Demographical, neuropsychological and behavioral task-fMRI characteristics

\begin{tabular}{lccc}
\hline & AD gr0 $(N=9)$ & AD gr1 $(N=15)$ & HC $(N=10)$ \\
\hline Age $(y)$ [Mean \pm SD] & $75.56 \pm 5.85$ & $74.67 \pm 5.39$ & $70.80 \pm 3.85$ \\
Range $(y)$ & $69-83$ & $61-81$ & $64-77$ \\
Male \% & 44.4 & 33.3 & 40.0 \\
MMSE score [mean \pm SD] & $19.37 \pm 3.24^{1}$ & $20.03 \pm 2.44^{2}$ & $28.31 \pm 1.8^{1,2}$ \\
Performance on fMRI task & $77.37 \pm 12.918^{1}$ & $80.14 \pm 4.378^{2}$ & $p<0.001$ \\
Accuracy & & $99.24 \pm 1.078^{1,2}$ & $p<0.001$ \\
\hline
\end{tabular}

Comparisons of MMSE and fMRI task variables between controls (HC) and patients with Alzheimer's disease (AD) were all significant at $p<0.001$; Post Hoc Test ${ }^{1} \mathrm{HC}$ versus AD gr0: $p<0.05{ }^{2} \mathrm{HC}$ versus AD gr1: $p<0.05$. AD gr1 = patients carried the rs363050 (AA or AG) and rs363043 (CT or TT) genotype; AD gr0= patients carried the rs363050 (GG) and rs363043 (CC) genotype. 

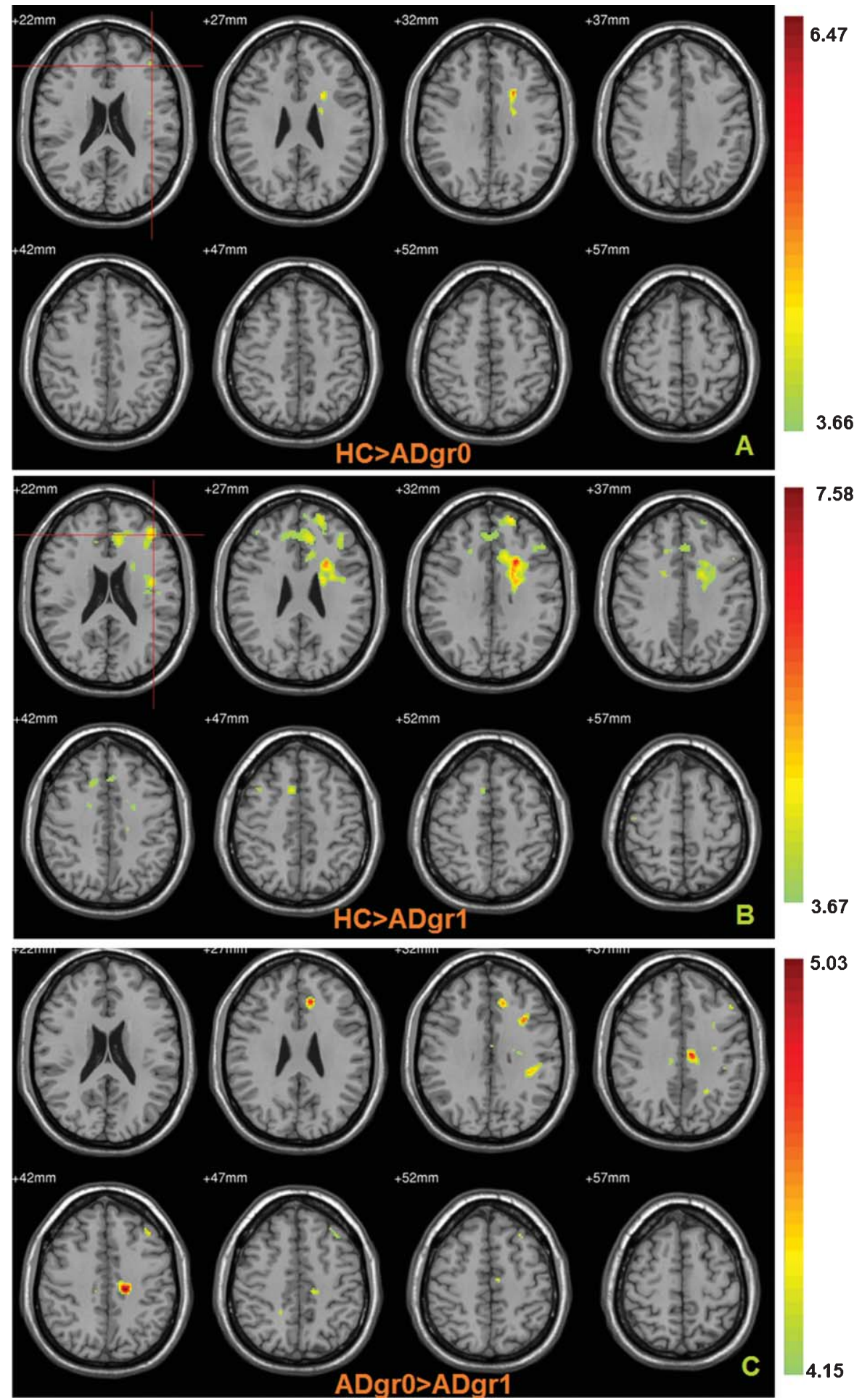

Fig. 2. Main BOLD effect due to the paced overt categorical verbal fluency task: comparison among the three groups: healthy controls (HC) (10 subjects), group 0 (10 AD patients carrying the rs363050 (GG) and rs363043 (CC) genotypes), and group 1 (18 AD patients carrying the rs363050 (AA or AG) and rs363043 (CT or TT) genotypes) (ANOVA). Brain areas which are significantly more activated in: $\mathrm{HC} 1$ versus AD group 0 (A); HC1 versus AD group 1 (B); and AD group 0 versus group 1 (panel C) are shown. See text for statistical thresholds and further details. The right side on the images corresponds to the left side of the brain. 
old to an uncorrected level for statistical purposes ( $\mathrm{p}_{\text {unc }}<0.001$ ), we noticed in AD patient compared to $\mathrm{HC}$ a significant hypoactivation also in temporal cortex (temporal pole and the fusiform gyrus). Nevertheless, direct comparison between the two AD groups (ANOVA) showed significant differences ( $\mathrm{p}_{\text {FWE-corr }}$ $<0.05$ at cluster level). Thus, brain activity was significantly reduced in group 1 patients in the cingulate cortex and in the frontal (middle and superior gyri) and the temporo- parietal (angular gyrus) area (Fig. 2C); in the opposite comparison (group $1>$ group 0 ) increased activation was not detected in any of the brain regions involved in the task.

\section{DISCUSSION}

SNAP-25 is a vesicular SNARE protein that plays an important role in the release of neurotransmitters via its interaction with voltage-gated calcium channels. SNAP-25 gene polymorphisms are suggested to associate with both variations in IQ phenotypes and a number of neurologic conditions, including the agerelated decline of cognitive function [44]. Because anatomical and functional synapsis alterations are present in $\mathrm{AD}$ we evaluated the possible involvement of SNAP-25 polymorphisms in this disease. SNAP-25 is a highly polymorphic gene as it includes 225 SNPs. We focused on those that had previously been shown to correlate with human diseases and variations in intelligence and that localize within intron 1 , in a region spanning about $13.8 \mathrm{~kb}$ known to affect transcription factor binding sites [18].

Results herein suggest that SNAP-25 gene polymorphism associate with $\mathrm{AD}$ and $\mathrm{aMCI}$ in Italian patients. Thus, the frequency of carriers of the SNAP-25 rs363050 (A)(AA/AG) and the rs363043 (T)(TT/CT) alleles was significantly increased in $\mathrm{AD}$ and in aMCI compared with gender- and age-matched healthy controls. Notably, a declining degree of prevalence of these alleles was present when $\mathrm{AD}$ (higher prevalence) were compared to aMCI (intermediate prevalence) and $\mathrm{HC}$ (lower prevalence); this observation is possibly due to the fact that not all aMCI will evolve into AD. It will be interesting to evaluate how many aMCI carrying the above-mentioned SNAP-25 genotypes will indeed develop AD.

As a result of the SNAP-25 allelic distribution, the rs363050/rs363043 A-T SNAP-25 haplotype was statistically more frequent in both $\mathrm{AD}$ and aMCI compared to HC. These two SNPs are in linkage disequilibrium, therefore their contribution may be due either to the fact that the rs363050 (A) genotype drags the rs363043 (T) genotype, or to the possibility that other genotypes within the haplotype are associated to AD development.

Having observed a possible association between particular SNAP-25 SNPs, AD, and aMCI, we next verified the presence of correlations between such SNPs and clinical parameters. Results showed that the SNAP-25 haplotypes more frequently seen in $\mathrm{AD}$, are associated with altered scores at the categorical fluency test. In particular, pathological mean scores in this test $(\leq 25)$ were associated with the rs363050 (AA/AG) and rs363043 (CT/TT) alleles in AD, with the strongest association being seen with rs363050 (AA/AG). These alterations were present in $\mathrm{AD}$ alone, as in aMCI, scores were higher than the pathological cut off of 25 . Results were analyzed next taking into account gender and $A P O E 4$ positivity by performing stratified analysis of different $S N A P-25$ polymorphisms in relationship with gender and with APOE4 positivity. Results indicated that the SNAP-25 SNPs-associated categorical fluency impairment is independent of both APOE4 and gender. Results of further multivariate logistic stepwise regression showed that, although both the rs363050 (A) and rs363043 (T) allele are associated with categorical fluency impairment, the role played by rs363050 (A) is stronger than that of $\mathrm{rs} 363043(\mathrm{~T})$.

Verbal fluency, and in particular Category fluency, is altered in $\mathrm{AD}[20,21]$, and a category fluency task is incorporated in the CERAD (Consortium to Establish a Registry for Alzheimer's Disease) protocol for the diagnosis and the clinical staging of AD [45, 46]. Category fluency relies on the structure of the semantic network, on the availability of sound lexical-semantic representations, and on the access to semantic knowledge, three facets of cognition that are affected in AD $[20,21]$. It seems thus biologically relevant that data herein suggest that impairments of these key components of language functioning are associated with the SNAP-25 SNPs that prevail in AD patients.

Possible anatomical relationships between SNAP25 SNPs and categorical fluency impairment were finally analyzed by fMRI in a subset of AD patients. We adopted the verbal fluency paradigm described by Basho and colleagues [39] to test language function. This fMRI task was chosen because it allows an appropriate response monitoring and a tight control over and reduced individual variability of task performance, making it suitable for the application in patients with cognitive deficits. Results showed that brain activation and brain area recruitment (prefrontal regions, parieto-temporal area, and cingulate cortex) are signif- 
icantly diminished in patients carrying the SNAP-25 SNPs that are more frequent in $\mathrm{AD}$ and correlate with pathological categorical fluency scores. This activation pattern could reflects pathologic alterations within critical nodes of the neural networks subserving working memory and attentional-executive functions [47, 48]. Recent studies have shown a relationship between reduced neural metabolism and poor performances in semantic memory tasks $[24,49,50]$. Our results are in line with these works, and especially with those focusing on the left temporoparietal and left prefrontal cortex $[48,49]$. The most impaired areas we found in $\mathrm{AD}$ were the frontal cortex and the anterior cingulated. It is proven that damage in these areas could reasonably influence the efficiency of attentive processing in task accomplishment. Finally, though at lower levels of significance, $\mathrm{AD}$ patients presented a hypoactivation also in temporal pole cortex, an area that is commonly considered as integral part of the semantic network [51], and fusiform gyrus. Interestingly, the impairment in the fusiform gyrus, an area that mediates between lateral semantic memory and medial episodic memory encoding networks, is coherent with recent evidence [52] showing that impairment in frontal and temporal areas involved in language network characterize the clinical evolution of AD.

Brain activation impairments were significantly more frequent in $\mathrm{AD}$ patients carrying the rs 363050 (AA/AG) and/or rs363043 (CT/TT) genotypes, and, on the other hand, an augmented brain activity was seen in bilateral parietal and frontal brain area and in the cingulate cortex of $\mathrm{AD}$ patients carrying the rs363050 (GG) and/or rs363043 (CC) compared to those carrying the rs363050 (AA/AG) and/or rs363043 (CT/TT) genotypes. The higher activation of group 0 could be attributable to some sort of residual compensatory mechanisms in frontal areas that accounts for a semantic deficit [53]. Interestingly, the left inferior parietal lobe and angular gyrus are considered to be crucial areas in the processing hierarchy underlying concept retrieval and conceptual integration. A damage of the left angular gyrus is responsible for a variety of cognitive impairments, such as, among others, anomia, sentence comprehension impairment and dementia [54]. Notably, our AD patients did not show differences in activations of bilateral anterior temporal lobe (ATL) and TP. This lack of activation in ATL and TP can be read as a index of impairment of these areas that reflects a connective degeneration in temporal cortices in AD. Coherent with present results are recent findings on $\mathrm{AD}$ population [52] that show a decrease in neuro plasticity of temporal lobes with the progression of the disease.
Categorical verbal fluency tests assess medial temporal lobe function [22] and are positively correlated with neurobiological hippocampal and parietal lobe neurochemical abnormalities in AD [55]. The SNAP25 gene is highly expressed in the hippocampus [56], a brain structure that plays a crucial role in semantic fluency performance [57]. Animal studies showed that the hippocampal SNAP-25 protein is involved in memory consolidation and long-term memory formation in rats $[58,59]$; additional results indicate that changes of hippocampal SNAP-25 expression contribute to agerelated decline of cognitive function [44]. It is therefore tempting to speculate that the SNPs described herein could influence the neuronal density and connectivity of the hippocampus, modulating synaptic plasticity and neurogenesis in the left hippocampus. Both the SNPs described herein, rs363050 (A/G) and rs363043 $(\mathrm{C} / \mathrm{T})$, localize within intron 1 in a region spanning about $13.8 \mathrm{~kb}$ which is known to affect transcription factor binding sites [18]. We analyzed the functional effect, of rs363050 and 363043 SNPs on transcriptional activity using luciferase a reporter gene assay. Our preliminary results showed that the rs 363050 (A) allele associates with a significantly higher $S N A P-25$ expression compared to the rs $363050(\mathrm{G})$ allele (Braida et al., unpublished results). This could be due to the impairment of binding of factors involved in the modulation of the SNAP-25 gene expression level or to the binding of other factors, different from the ones that recognize the sequence of the parental allele, acting as repressor. Both reduced and excessive SNAP-25 activity has been implicated in various disease states that involve cognitive dysfunctions such as attention deficit hyperactivity disorder, schizophrenia, and AD [14, 60-63]. An excess of SNAP-25 activity during adulthood was shown to be sufficient to mediate significant deficits in the memory formation process. Expression of SNAP25 in the adult dorsal hippocampus was also demonstrated to result in the dysregulation of memory consolidation machinery in this brain region [64]; finally, overexpression of SNAP-25 in cultured hippocampal neurons was associated with impaired synaptic transmission [65]. Altogether, these results suggest that an increased SNAP-25 level does impair synaptic maturation and/or neurotransmission. Moreover, as SNAP-25 is an age-related protein which is present in two isoforms, SNAP-25a which prevails in younger individuals, and SNAP-25b which takes over is seen in adults, contradictory results could be due to the fact that these isoforms may be differently regulated by the same gene polymorphisms. We have not yet obtained definite results on the possible correlation between SNAP-25 
polymoprphisms and splicing and miRNA expression; these analyses are undergoing in our laboratory

Further analysis and independent validation in additional cohorts will be required to assess the relevance of these variants in $\mathrm{AD}$ and to establish the possible predictive value of $S N A P-25$ polymorphisms in the evolution of this disease.

\section{ACKNOWLEDGMENTS}

Funding for this study was provided by 2011 Ricerca Corrente [Italian Ministry of Health and $5 \times 1000$, 2008] and Monzino Foundation. The funders had no role in study design, data collection and analysis, decision to publish, or preparation of the manuscript.

Authors' disclosures available online (http://www.jalz.com/disclosures/view.php?id=2312).

\section{SUPPLEMENTARY MATERIAL}

The supplementary table is available in the electronic version of this article.

\section{REFERENCES}

[1] Roses AD (1998) Alzheimer diseases: A model of gene mutations and susceptibility polymorphisms for complex psychiatric diseases. Am J Med Genet 81, 49-57.

[2] DeKosky ST, Scheff SW (1990) Synapse loss in frontal cortex biopsies in Alzheimer's disease: Correlation with cognitive severity. Ann Neurol 27, 457-464.

[3] Sørensen JB (2005) SNARE complexes prepare for membrane fusion. Trends Neurosci 28, 453-455.

[4] Südhof TC, Jahn R (1991) Proteins of synaptic vesicles involved in exocytosis and membrane recycling. Neuron $\mathbf{6}$, 665-677.

[5] Catterall WA, Few AP (2008) Calcium channel regulation and presynaptic plasticity. Neuron 59, 882-901.

[6] Pozzi D, Condliffe S, Bozzi Y, Chikhladze M, Grumelli C, Proux-Gillardeaux V, Takahashi M, Franceschetti S, Verderio C, Matteoli M (2008) Activity-dependent phosphorylation of Ser187 is required for SNAP-25-negative modulation of neuronal voltage-gated calcium channels. Proc Natl Acad Sci U $S$ A 105, 323-328.

[7] Condliffe SB, Corradini I, Pozzi D, Verderio C, Matteoli M (2010) Endogenous SNAP-25 regulates native voltage-gated calcium channels in glutamatergic neurons. J Biol Chem $\mathbf{2 8 5}$, 24968-24976.

[8] Thompson PM, Kelley M, Yao J, Tsai G, van, Kammen DP (2003) Elevated cerebrospinal fluid SNAP-25 in schizophrenia. Biol Psychiatry 53, 1132-1137.

[9] Bronk P, Deák F, Wilson MC, Liu X, Südhof TC, Kavalali ET (2007) Differential effects of SNAP-25 deletion on $\mathrm{Ca} 2+-d e p e n d e n t$ and $\mathrm{Ca} 2+-$ independent neurotransmission. J Neurophysiol 98, 794-806.

[10] Etain B, Dumaine A, Bellivier F, Pagan C, Francelle L, Goubran-Botros H, Moreno S, Deshommes J, Moustafa K,
Le Dudal K, Mathieu F, Henry C, Kahn JP, Launay JM, Mühleisen TW, Cichon S, Bourgeron T, Leboyer M, Jamain S (2010) A SNAP25 promoter variant is associated with earlyonset bipolar disorder and a high expression level in brain. Mol Psychiatry 15, 748-755.

[11] Faraone SV, Perlis RH, Doyle AE, Smoller JW, Goralnick JJ, Holmgren MA (2005) Molecular genetics of attentiondeficit/hyperactivity disorder. Biol Psychiatry 57, 1313-1323. Review.

[12] Corradini I, Verderio C, Sala M, Wilson MC, Matteoli M (2005) SNAP-25 in neuropsychiatric disorders. Ann N Y Acad Sci 1152, 93-99.

[13] Terracciano A, Sanna S, Uda M, Deiana B, Usala G, Busonero F, Maschio A, Scally M, Patriciu N, Chen WM, Distel MA Slagboom EP, Boomsma DI, Villafuerte S, Sliwerska E, Burmeister M, Amin N, Janssens AC, van Duijn CM, Schlessinger D, Abecasis GR, Costa PT Jr (2010) Genome-wide association scan for five major dimensions of personality. Mol Psychiatry 15, 647-656.

[14] Barr CL, Feng Y, Wigg K, Bloom S, Roberts W, Malone M, Schachar R, Tannock R, Kennedy JL (2000) Identification of DNA variants in the SNAP-25 gene and linkage study of these polymorphisms and attention-deficit hyperactivity disorder. Mol Psychiatry 5, 405-409.

[15] Feng Y, Crosbie J, Wigg K, Pathare T, Ickowicz A, Schachar R, Feng Y, Crosbie J, Wigg K, Pathare T (2005) The SNAP25 gene as a susceptibility gene contributing to attentiondeficit hyperactivity disorder. Mol Psychiatry 10, 998-1005, 973.

[16] Guerini FR, Bolognesi E, Chiappedi M, Manca S, Ghezzo A, Agliardi C, Sotgiu S, Usai S, Matteoli M, Clerici M (2011) SNAP-25 single nucleotide polymorphisms are associated with hyperactivity in autism spectrum disorders. Pharmacol Res 64, 283-288.

[17] Postuma D, Luciano M, Geus EJ, Wright MJ, Slagboom PE, Montgomery GW, Boomsma DI, Martin NG (2005) A genomewide scan for intelligence identifies quantitative trait loci on 2q and 6p. Am J Hum Genet 77, 318-326.

[18] Gosso MF, de Geus EJ, Polderman TJ, Boomsma DI, Heutink P, Posthuma D (2008) Common variants underlying cognitive ability: Further evidence for association between the SNAP25 gene and cognition using a family-based study in two independent Dutch cohorts. Genes Brain Behav 7, 355-364.

[19] Verma M, Howard RJ (2012) Semantic memory and language dysfunction in early Alzheimer's disease: A review. Int J Geriatr Psychiatry 27, 1209-1217.

[20] Henry JD, Crawford JR, Phillips LH (2004) Verbal fluency performance in dementia of the Alzheimer's type: A metaanalysis. Neuropsychologia 42, 1212-1222.

[21] Capitani E, Rosci C, Saetti MC, Laiacona M (2009) Mirror asymmetry of Category and Letter fluency in traumatic brain injury and Alzheimer's patients. Neuropsychologia 47, 423429.

[22] Pihlajamäki M, Tanila H, Hänninen T, Könönen M, Laakso M, Partanen K, Soininen H, Aronen HJ (2000) Verbal fluency activates the left medial temporal lobe: A functional magnetic resonance imaging study. Ann Neurol 47, 470-476.

[23] Grossman M, Koenig P, Glosser G, DeVita C, Moore P, Rhee J, Detre J, Alsop D, Gee J (2003) Neural basis for semantic memory difficulty in Alzheimer's disease: An fMRI study. Brain 126, 292-311.

[24] Zahn R, Garrard P, Talazko J, Gondan M, Bubrowski P, Juengling F, Slawik H, Dykierek P, Koester B, Hull M (2006) Patterns of regional brain hypometabolismassociated with knowledge of semantic features and categories in Alzheimer's disease. J Cogn Neurosci 18, 2138-2151. 
[25] McKhann G, Drachman D, Folstein M, Katzman R, Price D, Stadlan EM (1984) Clinical diagnosis of Alzheimer's disease: Report of the NINCDS-ADRDA Work Group under the auspices of Department of Health and Human Services Task Force on Alzheimer's Disease. Neurology 34, 939-944.

[26] American Psychiatric Association (2000) Diagnostic and Statistical Manual of Mental Disorders, 4th ed. Text Revision. American Psychiatric Press, Washington, DC.

[27] Petersen RC (2004) Mild cognitive impairment as a diagnostic entity. J Intern Med 256, 183-194.

[28] Morris JC (1993) The Clinical Dementia Rating (CDR): Current version and scoring rules. Neurology $\mathbf{4 3}, 2412-2414$.

[29] Rosen WG, Terry RD, Fuld PA, Katzman R, Peck A (1980) Pathological verification of ischemic score in differentiation of dementias. Ann Neurol 7, 486-488.

[30] Hamilton M (1960) A rating scale for depression. J Neurol Neurosurg Psychiatry 23, 56-62.

[31] Grundman M, Petersen RC, Ferris SH, Thomas RG, Aisen PS, Bennett DA, Foster NL, Jack CR Jr, Galasko DR, Doody R, Kaye J, Sano M, Mohs R, Gauthier S, Kim HT, Jin S, Schultz AN, Schafer K, Mulnard R, van Dyck CH, Mintzer J, Zamrini EY, Cahn-Weiner D, Thal LJ, Alzheimer's Disease Cooperative, Study (2004) Mild cognitive impairment can be distinguished from Alzheimer disease and normal aging for clinical trials. Arch Neurol 61, 59-66.

[32] Ligthart GJ, Corberand JX, Fournier C, Galanaud P, Hijmans W, Kennes B, Müller-Hermelink HK, Steinmann GG (1984) Admission criteria for immunogerontological studies in man The SENIEUR protocol. Mech Ageing Dev 28, 47-55.

[33] Speciale S, Bellelli G, Lucchi E, Trabucchi M (2007) Delirium and functional recovery in elderly patients. J Gerontol A Biol Sci Med Sci 62, 107-108; author reply 108.

[34] Magni E, Binetti G, Bianchetti A, Rozzini R, Trabucchi M (1996) Mini-Mental State Examination: A normative study in Italian elderly population. Eur J Neurol 3, 198-202.

[35] Novelli G, Papagno C, Capitani E, Laiacona M, Vallar G, Cappa SF (1986) Tre test di ricerca e produzione lessicale: Taratura su soggetti normali. Archo Psicol Neurol Psichiat 47, 477-506.

[36] Spinnler H, Tognoni P (1987) Standardizzazione e taratura italiana di test neuropsicologici. Ital J Neurol Sci 86 , $1-20$.

[37] Bertolani L, De Renzi E, Faglioni P (1993) Test di memoria non verbale di impiego diagnostico in clinica: Taratura su soggetti normali. Archo Psicol Neurol Psichiat 54, 477-486.

[38] Raven JC, Lewis HK (1971) Coloured progressive matrices sets, Psychological Corporation, London.

[39] Basho S, Palmer ED, Rubio MA, Wulfeck B, Müller RA (2007) Effects of generation mode in fMRI adaptations of semantic fluency: Paced production and overt speech. Neuropsychologia 45, 1697-1706

[40] Shi YY, He L (2005) SHEsis, a powerful software platform for analyses of linkage disequilibrium, haplotype construction, and genetic association at polymorphism loci. Cell Res $\mathbf{1 5}$ 97-98.

[41] Li Z, Zhang Z, He Z, Tang W, Li T, Zeng Z, He L, Shi Y (2009) A partition-ligation-combination-subdivision EM algorithm for haplotype inference with multiallelic markers: Update of the SHEsis (http://analysis.bio-x.cn). Cell Res 19, 519-523.

[42] Purcell S, Neale B, Todd-Brown K, Thomas L, Ferreira MA Bender D, Maller J, Sklar P, de Bakker PI, Daly MJ, Sham PC (2007) PLINK: A tool set for whole-genome association and population-based linkage analyses. Am J Hum Genet $\mathbf{8 1}$ $559-575$.
[43] Friston KJ, Holmes AP, Poline JB, Grasby PJ, Williams SC, Frackowiak RS, Turner L (1995) Analysis of fMRI time-series revisited. Neuroimage 2, 45-53.

[44] Cao L, Wang F, Yang QG, Jiang W, Wang C, Chen YP, Chen GH (2012) Reduced thyroid hormones with increased hippocampal SNAP-25 and Munc18-1 might involve cognitive impairment during aging. Behav Brain Res 229, 131-137.

[45] Monsch AU, Bondi MW, Butters N, Salmon DP, Katzman R, Thal LJ (1992) Comparisons of verbal fluency tasks in the detection of dementia of the Alzheimer type. Arch Neurol 49, 1253-1258.

[46] Welsh KA, Butters N, Huges JP, Mohs RC, Heyman A (1992) Detection and staging of dementia in Alzheimer's disease: Use of the neuropsychological measures developed for the Consortium to Establish a Registry for Alzheimer's disease. Arch Neurol 49, 448-452.

[47] Smith EE, Jonides J, Marshuetz C, Koeppe RA (1998) Components of verbal working memory: Evidence from neuroimaging. Proc Natl Acad Sci U S A 95, 876-882.

[48] Wagner AD (1999) Working memory contributions to human learning and remembering. Neuron 22, 19-22.

[49] Teipel SJ, Willoch F, Ishii K, Bürgera K, Drzezgab A, Engeld R, Bartensteine P, Möllera H-J, Schwaigerb M, Hampela H (2006) Resting state glucose utilization and the CERAD cognitive battery in patients with Alzheimer's disease. Neurobiol Aging 27, 681-690.

[50] Morris JC, Balota DA (2001) Semantic dementia versus Alzheimer's disease: A matter of semantics? Neurology 57, 173-174.

[51] Binder JR, Desai RH, Graves WW, Conant LL (2009) Where is the semantic system? A critical review and meta-analysis of 120 functional neuroimaging studies. Cereb Cort 19, 27672796.

[52] Domoto-Reilly K, Sapolsky D, Brickhouse M, Dickerson BC, the Alzheimer's Disease Neuroimaging, Initiative (2012) Naming impairment in Alzheimer's disease is associated with left anterior temporal lobe atrophy. NeuroImage 63, 348-355.

[53] Preti MG, Makris N, Papadimitriou G, Laganà MM, Griffanti L, Clerici M, Nemni R, Westin CF, Baselli G, Baglio F (2014) A novel approach of groupwise fMRI-guided tractography allowing to characterize the clinical evolution of Alzheimer's disease. PLoS One $\mathbf{9}$, e92026.

[54] Seidenberg M, Guidotti L, Nielson KA, Woodard JL, Durgerian S, Antuono P, Zhang Q, Rao SM (2009) Semantic memory activation in individuals at risk for developing Alzheimer's disease. Neurology 73, 612-620.

[55] Ackl N, Ising M, Schreiber YA, Atiya M, Sonntag A, Auer DP (2005) Hippocampal metabolic abnormalities in mild cognitive impairment and Alzheimer's disease. Neurosci Lett 384, 23-28.

[56] Geddes JW, Hess EJ, Hart RA, Kesslak JP, Cotman CW, Wilson MC (1999) Lesions of hippocampal circuitry define synaptosomal-associated protein-25 (SNAP-25) as a novel presynaptic marker. Neuroscience $\mathbf{3 8}, 515-525$.

[57] Gleissner U, Elger CE (2001) The hippocampal contribution to verbal fluency in patients with temporal lobe epilepsy. Cortex 37, 55-63.

[58] Hou Q, Gao X, Zhang X, Kong L, Wang X, Bian W, Tu Y, Jin M, Zhao G, Li B, Jing N, Yu L (2004) SNAP-25 in hippocampal CA1 region is involved in memory consolidation. Eur J Neurosci 20, 1593-1603.

[59] Hou QL, Gao X, Lu Q, Zhang XH, Tu YY, Jin ML, Zhao GP, Yu L, Jing NH, Li BM (2006) SNAP-25 in hippocampal CA3 region is required for long-term memory formation. Biochem Biophys Res Commun 47, 955-962. 
[60] Greber S, Lubec G, Cairns N, Fountoulakis M (1999) Decreased levels of synaptosomal associated protein 25 in the brain of patients with Down syndrome and Alzheimer's disease. Electrophoresis 20, 928-934.

[61] Mukaetova-Ladinska EB, Hurt J, Honer WG, Harrington CR, Wischik CM (2002) Loss of synaptic but not cytoskeletal proteins in the cerebellum of chronic schizophrenics. Neurosci Lett 317, 161-165.

[62] Mill J, Richards S, Knight J, Curran S, Taylor E, Asherson P (2004) Haplotype analysis of SNAP-25 suggests a role in the aetiology of ADHD. Mol Psychiatry 9, 801-810.

[63] Jeans AF, Oliver PL, Johnson R, Capogna M, Vikman J, Molnár Z, Babbs A, Partridge CJ, Salehi A, Bengtsson M, Eliasson L, Rorsman P, Davies KE (2007) A dominant mutation in Snap25 causes impaired vesicle trafficking, sensorimotor gating, and ataxia in the blind-drunk mouse. Proc Natl Acad Sci U S A 104, 2431-2436.

[64] McKee AG, Loscher JS, O'Sullivan NC, Chadderton N, Palf A, Batti L, Sheridan GK, O'Shea S, Moran M, McCabe O, Fernández AB, Pangalos MN, O’Connor JJ, Regan CM, O'Connor WT, Humphries P, Farrar GJ, Murphy KJ (2010) AAV-mediated chronic over-expression of SNAP-25 in adult rat dorsal hippocampus impairs memory-associated synaptic plasticity. J Neurochem 112, 991-1004.

[65] Owe-Larsson B, Berglund M, Kristensson K, Garoff H, Larhammar D, Brodin L, Low P (1999) Perturbation of the synaptic release machinery in hippocampal neurons by overexpression of SNAP-25 with the Semliki Forest virus vector. Eur J Neurosci 11, 1981-1987. 